Supplement of Hydrol. Earth Syst. Sci., 21, 2843-2861, 2017

https://doi.org/10.5194/hess-21-2843-2017-supplement

(c) Author(s) 2017. This work is distributed under

the Creative Commons Attribution 3.0 License.

(c) (1)

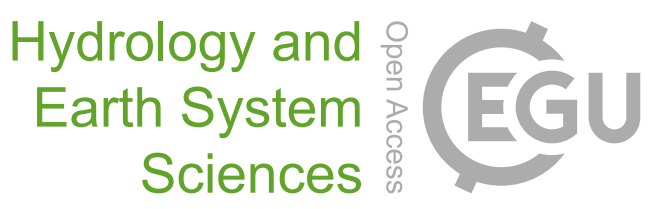

Supplement of

\title{
Land surface model performance using cosmic-ray and point-scale soil moisture measurements for calibration
}

Joost Iwema et al.

Correspondence to: Joost Iwema (joost.iwema@ hotmail.com)

The copyright of individual parts of the supplement might differ from the CC BY 3.0 License. 


\section{Supplement 1: Methodology figures}

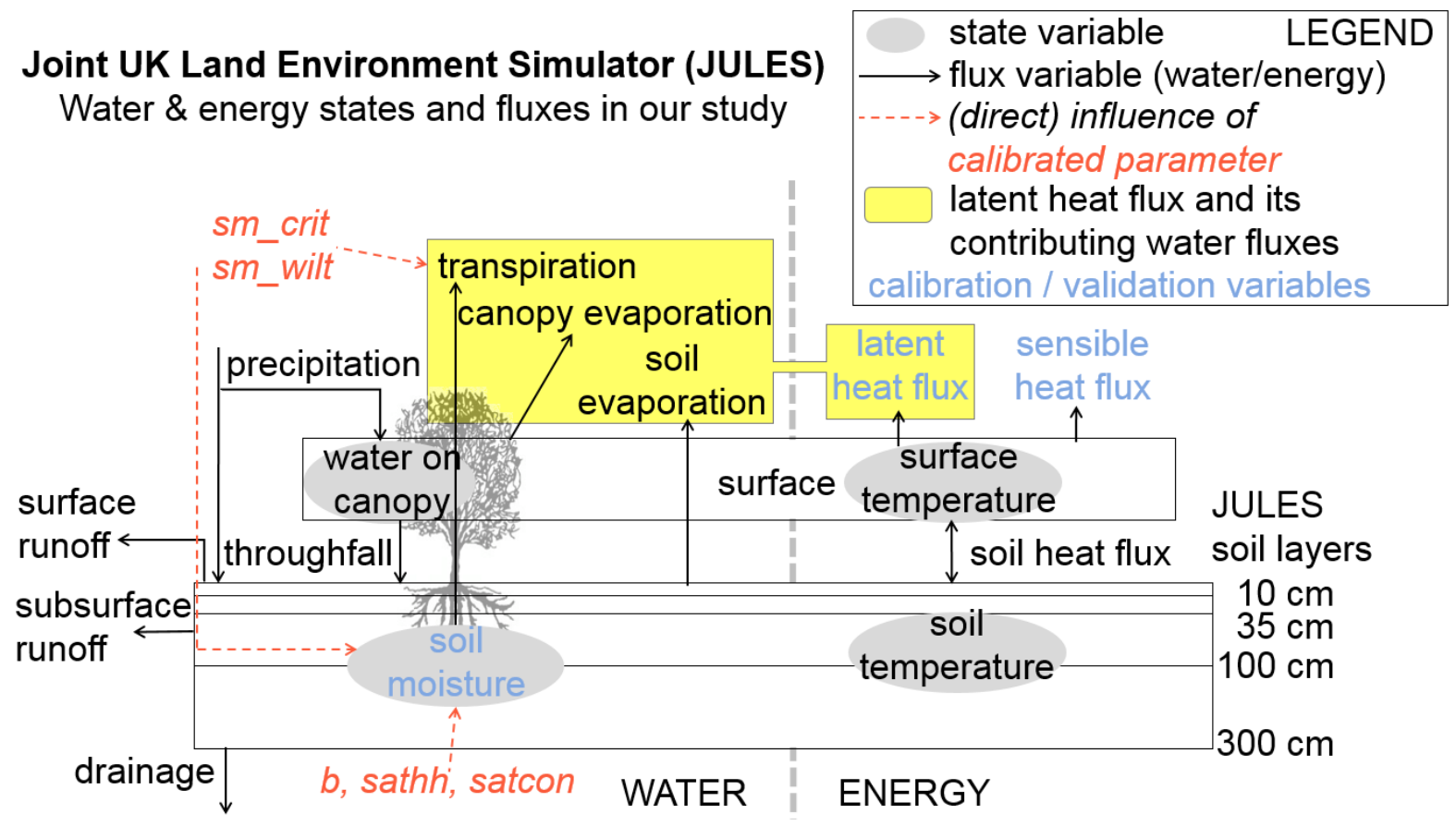

Figure S1.1: JULES water and energy states and fluxes most relevant to our study. Calibrated parameters and their direct effects shown. Please, refer to Table 2 for detailed description of parameters. 


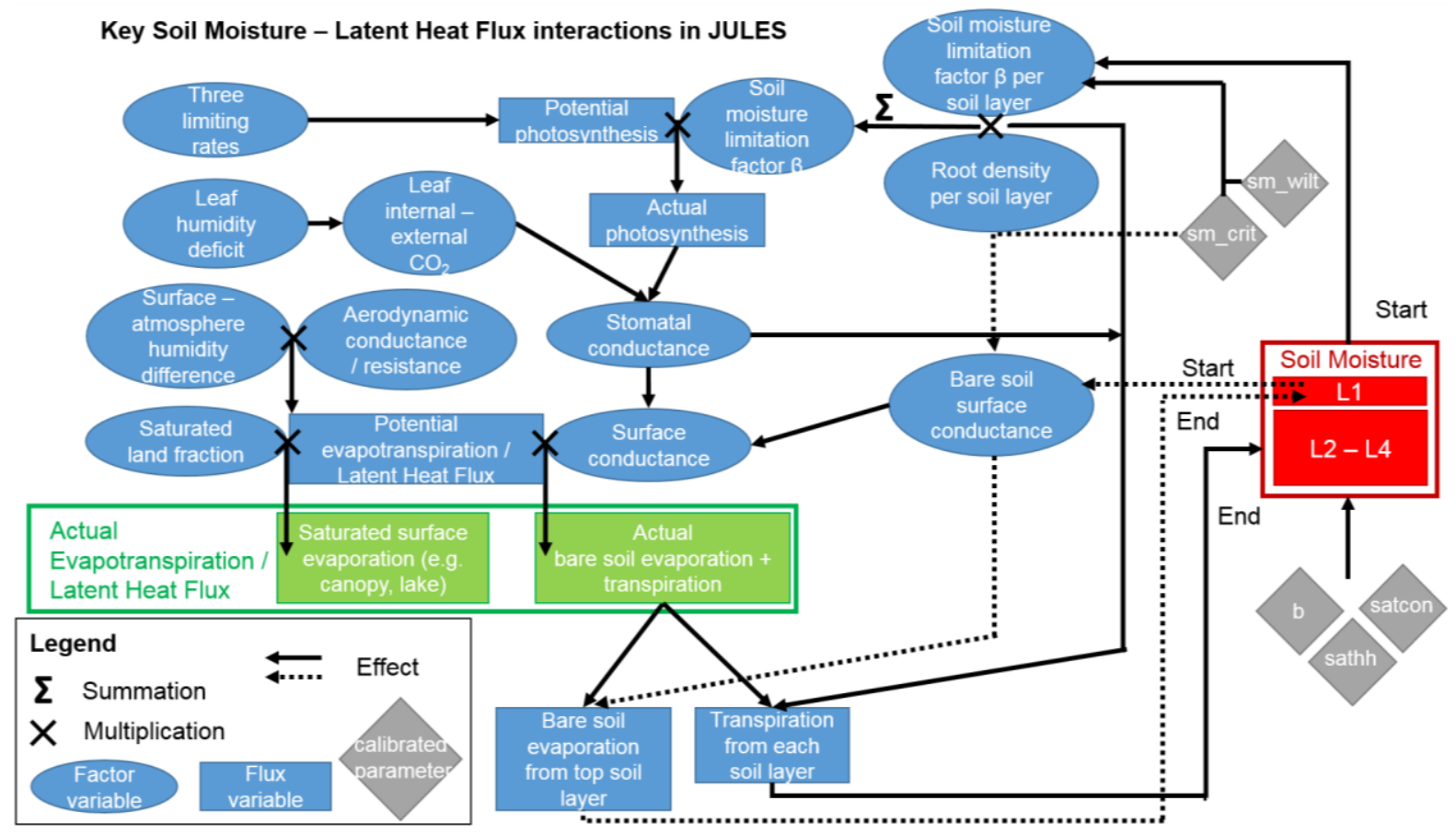

Figure S1.2: Key interactions between soil moisture and latent heat flux in JULES. The parameters calibrated in this study are indicated. 


\section{Supplement 2: Evaporative Fraction results}

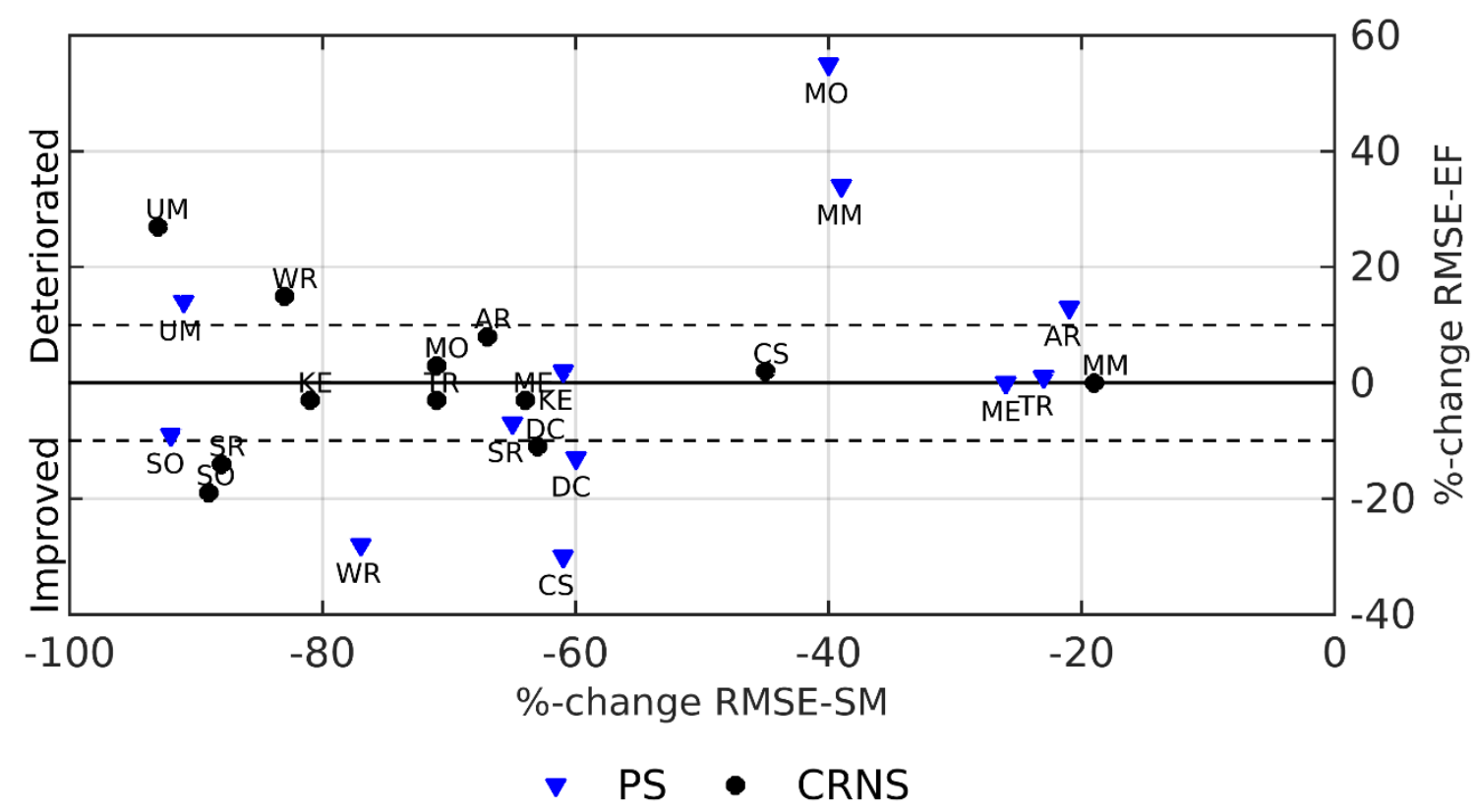

Figure S2.1: Root Mean Square Error (RMSE) values between observed and simulated evaporative fraction $(\mathrm{EF}=\mathrm{LE} /(\mathrm{LE}+\mathrm{H}))$ plotted against RMSE values between observed and simulated soil moisture, per site, for default and single-objective calibrated (PS and CRNS) runs.

\section{Supplement 3: Parameter values}

If parameter values obtained after calibration were not physically feasible (e.g. representing a sandy soil while there was a clay soil) then, if model structure is assumed to represent biophysical processes sufficiently well, that could yield undesirable results. We investigated whether the parameter values obtained were 'realistic' by seeing if they were substantially different from the soil conditions reported by HWSD (used to obtain default values), COSMOS, and Ameriflux. We analysed the calibrated parameter values from the single-objective calibrations for each site with the parallel coordinate plots in Figure S3.1. Calibration yielded values far from default for sensitive parameter $\mathrm{b}$ in two cases (MM-PS and TR-PS) only. The saturation hydraulic conductivity took on substantially different values in eight cases. The critical point and wilting point soil moisture

15 parameters assumed values far from the defaults in three and four cases respectively. The wilting point multiplier, which was actually calibrated, took a wide range of values, but the actual wilting point parameter stayed closer to the default values in most cases.

Although the parameter calibration range of parameter sathh was rather wide compared to the range of soil types from HWSD at the twelve sites, the values were within the boundaries at all sites for the default runs and in thirteen cases after the 
calibrations. The saturated hydraulic conductivity was within the edges for 22 runs. Especially for the saturation hydraulic conductivity, the parameter calibration range was non-linear, with a factor 1000 difference between the upper and lower boundaries.

COSMOS/Ameriflux soil information (Table 1 of the manuscript) for UM suggests sandier conditions, which would yield

5 higher saturation hydraulic conductivity, which was indeed obtained after PS calibration. At SO calibration yielded higher values for the saturation hydraulic conductivity and a lower value for $b$, which is in line with the thick organic layer occurring there. At the KE site, the soil was reported to be coarser than actually reported by the HWSD, and also to contain stones. This would mean higher saturation hydraulic conductivity, but a higher value was not obtained after calibration. At SR and MM the soil was reported to be of finer texture than the data from HWSD. Lower saturation hydraulic conductivity values than the

10 defaults were however not obtained. At TR a clay hardpan was found at 30-40 cm, which would impede drainage and calibration could hence be expected to yield lower saturation hydraulic conductivity. This did however not happen after our calibrations. AR and WR were reported to be sandy, in contrast with the loam soils reported by HWSD. Higher saturation hydraulic conductivity values were found after CRNS calibration at AR and after both calibrations at WR.

Several reasons have been proposed why apparently non-physically realistic parameter values were found, of which we discuss

15 the most plausible ones here. Richards' Equation applicability at larger horizontal scales is questionable for multiple reasons (Beven and Germann, 2013). The foremost reason is hydraulic continuity, upon which Richards' Equation is based, does usually not occur in the field over distances of multiple metres. This is due to horizontal and vertical soil heterogeneity (e.g. macro-pores, soil layering in organic matter and particle sizes, stones) not (properly) accounted for in the model. The form of Richards' Equation implemented in JULES does not take these natural features into account and therefore effective parameters

20 can be expected to differ from values representing a soil with a homogeneous matrix only. That means the process represented by the equation is not the same as the processes occurring in the field soil. Therefore the parameters' roles in JULES differ from their theoretically assumed roles, and so different effective parameter values can be expected. Explicitly taking into account heterogeneity issues like macro-pores could improve JULES' performance (Rahman and Rosolem, 2017), but doing so was beyond the scope of our study. The vertical discretisation of the soil (layers of 10, 25, 65, and $200 \mathrm{~cm}$ ) may not be

25 suitable for solving Richards' Equation. Layer thicknesses of no more than one centimetre near the surface are required to accurately simulate water fluxes. Insufficiently fine discretisation at both field ( eddy-covariance footprint) and hydrological catchment scale may not yield realistic water fluxes using realistic parameter values. (Smirnova et al., 1997; Lee and Abriola, 1999; Van Dam and Feddes, 2000; Downer and Ogden, 2004; Beven and Germann, 2013). Testing the effects of this issue on our results, which would entail changing the vertical discretisation of the soil column in JULES, was beyond the scope of our 30 study.

Finally, due to the simplifications of natural processes inherent to models, physically realistic parameters often provide unrealistic results (Gupta et al., 1998). 

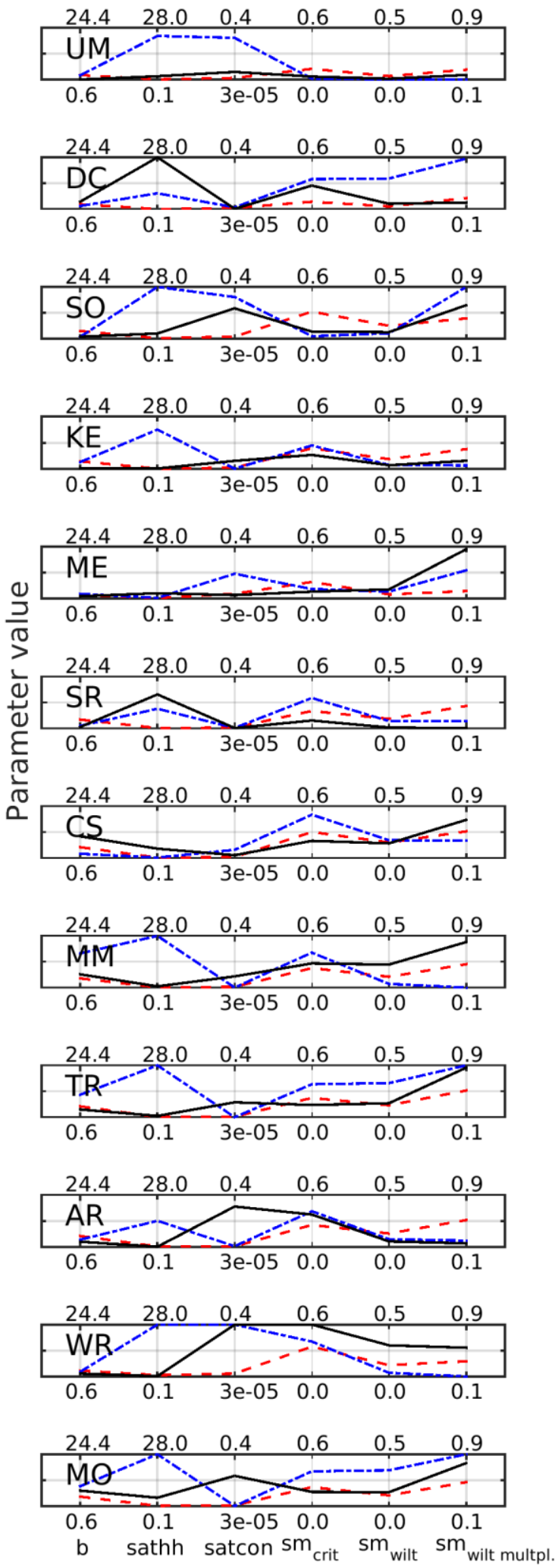
Figure S3.1: Parameter values of the Default, PS-calibrated, and CRNS-calibrated solutions for each site shown in parallel coordinate plots. Parameter b is Mualem-Van Genuchten parameter 1/(n-1), sathh is Mualem-Van Genuchten parameter $\alpha^{-1}$, satcon is the saturated soil hydraulic conductivity in the Mualem function, $\mathbf{s m}_{\text {crit }}$ is the critical point soil moisture content, $\mathbf{s m}_{\text {wilt }}$ is the wilting point soil moisture content, and is the multiplier (multiplied with $\mathbf{s m}_{\text {crit}}$ ) which was calibrated instead of smwilt. The upper

5 and lower bound values of the calibration ranges are shown for each parameter and were the same for all sites

\section{References}

Beven, K. and Germann, P.: Macropores and water flow in soils revisited, Water Resour. Res., 49, 3071-3092,

10 https://doi.org/10.1002/wrcr.20156, 2013.

Downer, C. W. and Ogden, F. L.: Appropriate vertical discretization of Richards' equation for two-dimensional watershedscale modelling, Hydrol. Process., 18, 1-22, 2004.

Smirnova, T. G., Brown, J. M., and Benjamin, S. G.: Performance of temperature and surface fluxes, Mon. Weather Rev., 15 125, 1871-1884, 1997.

Van Dam, J. C. and Feddes, R. A.: Numerical simulation of infiltration, evaporation, and shallow groundwater levels with the Richards equation, J. Hydrol., 233, 72-85, 2000. 